Research Article

\title{
New Treatment of the Rotary Motion of a Rigid Body with Estimated Natural Frequency
}

\author{
A. I. Ismail $\mathbb{D}^{1,2}$ \\ ${ }^{1}$ Mechanical Engineering Department, College of Engineering and Islamic Architecture, Umm Al-Qura University, \\ P.O. Box 5555, Makkah, Saudi Arabia \\ ${ }^{2}$ Mathematics Department, Faculty of Science, Tanta University, Tanta, P.O. Box 31527, Egypt
}

Correspondence should be addressed to A. I. Ismail; aiismail@uqu.edu.sa

Received 6 October 2020; Revised 3 November 2020; Accepted 6 November 2020; Published 7 December 2020

Academic Editor: Juan L. G. Guirao

Copyright $\odot 2020$ A. I. Ismail. This is an open access article distributed under the Creative Commons Attribution License, which permits unrestricted use, distribution, and reproduction in any medium, provided the original work is properly cited.

\begin{abstract}
In this paper, the problem of the motion of a rigid body about a fixed point under the action of a Newtonian force field is studied when the natural frequency $\omega=0.5$. This case of singularity appears in the previous works and deals with different bodies which are classified according to the moments of inertia. Using the large parameter method, the periodic solutions for the equations of motion of this problem are obtained in terms of a large parameter, which will be defined later. The geometric interpretation of the considered motion will be given in terms of Euler's angles. The numerical solutions for the system of equations of motion are obtained by one of the well-known numerical methods. The comparison between the obtained numerical solutions and analytical ones is carried out to show the errors between them and to prove the accuracy of both used techniques. In the end, we obtain the case of the regular precession type as a special case. The stability of the motion is considered by the phase diagram procedures.
\end{abstract}

\section{Introduction}

Consider a rigid body of mass $M$ moves in an asymmetric field around a fixed point $O$ [1]. Let us assume that the surface of its ellipsoid of inertia is optional, as well as the mass center. Let the frame $O X, O Y$, and $O Z$ be a fixed system in space, and the frame $O x, O y$, and $O z$ is the main axes frame for the surface of the ellipsoid of inertia of the body which moves with the it. Initially, we consider the main axis $z$ for the surface of the ellipsoid of inertia that makes an angle $\xi_{0} \neq(k \pi / 2)$; $k=0,1$, and 2 with the fixed axis $Z$ in space. Let the body spins with small speed angular velocity $r_{0}$ about the axis $z$. Suppose that $p, q$, and $r$ represent the components of the angular velocity vector of the body about the main axes of the ellipsoid of the inertia surface; $\gamma, \gamma^{\prime}$, and $\gamma^{\prime \prime}$ are the directional cosines vector of the axis $Z ; g$ is the acceleration of gravity; $A, B$, and $C$ are the principal moments of inertia. The point $\left(x_{0}, y_{0}, z_{0}\right)$ is the center of mass in the moving coordinate system; $\underline{R}$ is the position vector of the center of attraction $0_{1}$ on the fixed downward coordinate $Z$ axis, and $\rho$ is the position vector of the element $\mathrm{d} m$. Let $\underline{\hat{i}}, \hat{j}, \underline{\underline{k}}$, and $\underline{\underline{Z}}$ be the unit vectors in the shown directions (Figure 1). Consider $\mathrm{d} \underline{F}$ is the attraction force element due to the attracting center and acted on the element $\mathrm{d} m$ at the point $p(x, y, z)$.

\section{Formulation of the Problem}

Without a loss of generality, we choose the positive direction of both the axis $z$ and the axis $x$ that do not make an obtuse angle $\xi_{0}$ with the direction of axis $Z$. Under the restriction on $\xi_{0}$ and the choice of the coordinate system, we get [2]

$$
\gamma_{0} \geq 0, \quad 0<\gamma_{0}^{\prime \prime}<1 .
$$

The differential equations of motion can be reduced to an autonomous system of two degrees of freedom and one first integral as follows [3]:

$$
\begin{gathered}
4 \ddot{p}_{2}+p_{2}=4 \varepsilon^{-2} F\left(p_{2}, \dot{p}_{2}, \gamma_{2}, \dot{\gamma}_{2}, \varepsilon\right), \\
\ddot{\gamma}_{2}+\gamma_{2}=\varepsilon^{-2} \Phi\left(p_{2}, \dot{p}_{2}, \gamma_{2}, \dot{\gamma}_{2}, \varepsilon\right), \\
\gamma_{2}^{2}+\dot{\gamma}_{2}^{2}+2 \varepsilon^{-1}\left(\nu p_{2} \gamma_{2}+v_{2} \dot{p}_{2} \dot{\gamma}_{2}+s_{21}\right)+\varepsilon^{-2}(\ldots)=\gamma_{0}^{\prime \prime-2}-1,
\end{gathered}
$$




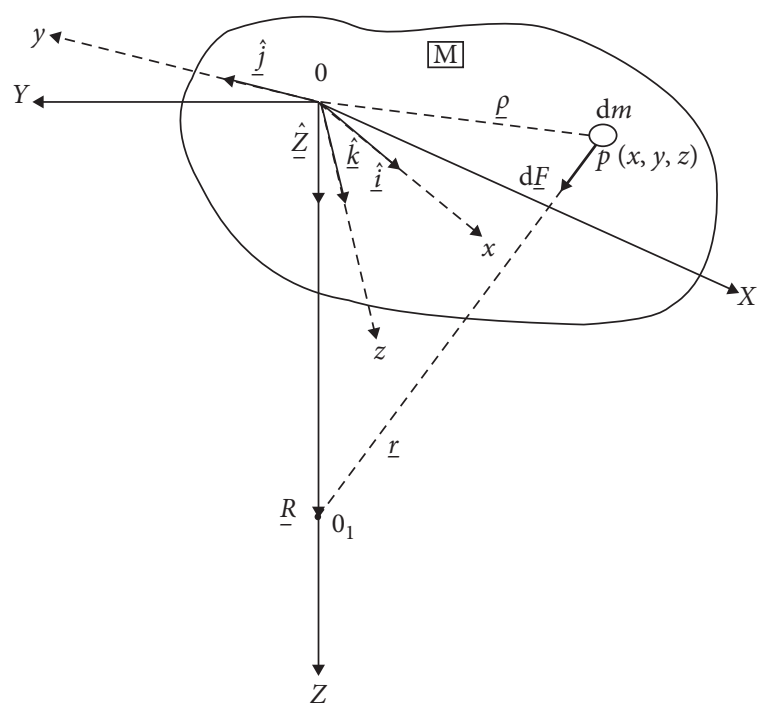

Figure 1: Description of motion in terms of moving and fixed frames.

where

$$
\begin{aligned}
F= & C_{1} A_{1}^{-1} p_{2} \dot{p}_{2}^{2}+x_{0}^{\prime} \dot{p}_{2} \dot{\gamma}_{2}-y_{0}^{\prime} a^{-1} p_{2} \dot{\gamma}_{2} \\
& -y_{0}^{\prime} A_{1}^{-1}\left(A_{1}+a^{-1}\right) \gamma_{2} \dot{p}_{2}-z_{0}^{\prime} a^{-1} p_{2} \\
& -0.75 v e_{1} p_{2}-0.25 p_{2} s_{11}+A_{1} b^{-1} x_{0}^{\prime} s_{21}+O\left(\varepsilon^{-1}\right)+\cdots, \\
\Phi= & -\left(1-C_{1}\right) A_{1}^{-1} p_{2} \dot{p}_{2} \dot{\gamma}_{2}+x_{0}^{\prime} \dot{\gamma}_{2}^{2}-y_{0}^{\prime} \gamma_{2} \dot{\gamma}_{2}-z_{0}^{\prime} b^{-1} \gamma_{2} \\
& +x_{0}^{\prime} b^{-1}-A_{1}^{-2} \gamma_{2} \dot{p}_{2}^{2} \\
& +0.75 \nu\left(e+e_{1} \gamma_{2}\right)-\gamma_{2} s_{11} \\
& +\left(1+B_{1}\right) p_{2} s_{21}+O\left(\varepsilon^{-1}\right)+\cdots,
\end{aligned}
$$

$$
\begin{aligned}
p_{2}= & p_{1}-\varepsilon^{-1}\left(e+e_{1} \gamma_{2}\right) \\
\gamma_{2}= & \gamma_{1}-\varepsilon^{-1} \nu p_{2}, \\
q_{1}= & -A_{1}^{-1} \dot{p}_{2}+\varepsilon^{-1} A_{1}^{-1}\left(y_{0}^{\prime} a^{-1}-e_{2} \dot{\gamma}_{2}\right)+\cdots \\
r_{1}= & 1+0.5 \varepsilon^{-2} s_{11}+\cdots, \\
\gamma_{1}^{\prime}= & \dot{\gamma}_{2}+\varepsilon^{-1} \nu_{2} \dot{p}_{2}+\cdots, \\
\gamma_{1}^{\prime \prime}= & 1+\varepsilon^{-1} s_{21}+\varepsilon^{-2}\left(s_{22}-0.5 s_{11}\right)+\cdots, \\
p_{1}= & \frac{p}{c} \sqrt{\gamma_{0}^{\prime \prime}}, \\
& \cdot(p q) \\
r_{1}= & \frac{r}{r_{0}}, \\
\gamma_{1}= & \frac{\gamma}{\gamma_{0}^{\prime \prime}}, \\
& \cdot\left(\gamma \gamma^{\prime} \gamma^{\prime \prime}\right) \\
\tau= & r_{0}^{-1} t, \\
& \cdot\left(. \equiv \frac{\mathrm{d}}{\mathrm{d} \tau}\right)
\end{aligned}
$$

$$
\begin{aligned}
s_{11}= & \frac{a\left(p_{20}^{2}-p_{2}^{2}\right)+b\left(\dot{p}_{20}^{2}-\dot{p}_{2}^{2}\right)}{A_{1}^{2}-2\left[x_{0}^{\prime}\left(\gamma_{20}-\gamma_{2}\right)+y_{0}^{\prime}\left(\dot{\gamma}_{20}-\dot{\gamma}_{2}\right)\right]}, \\
s_{21}= & a\left(p_{20} \gamma_{20}-p_{2} \gamma_{2}\right)-b A_{1}^{-1}\left(\dot{p}_{20} \dot{\gamma}_{20}-\dot{p}_{2} \dot{\gamma}_{2}\right), \\
s_{22}= & a\left[\nu\left(p_{20}^{2}-p_{2}^{2}\right)+e\left(\gamma_{20}-\gamma_{2}\right)+e_{1}\left(\gamma_{20}^{2}-\gamma_{2}^{2}\right)\right] \\
& +b A_{1}^{-1}\left[-v_{2}\left(\dot{p}_{20}^{2}-\dot{p}_{2}^{2}\right)+a^{-1} y_{0}^{\prime}\left(\dot{\gamma}_{20}-\dot{\gamma}_{2}\right)-e_{2}\left(\dot{\gamma}_{20}^{2}-\dot{\gamma}_{2}^{2}\right)\right],
\end{aligned}
$$

$$
A_{1}=\frac{C-B}{A}
$$$$
(A B C) \text {, }
$$$$
a=\frac{A}{C}
$$$$
(a b) \text {, }
$$$$
c^{2}=\frac{M g l}{C},
$$$$
\varepsilon=\frac{c \sqrt{\gamma_{0}^{\prime \prime}}}{r_{0}},
$$$$
x_{0}=l x_{0}^{\prime} \text {, }
$$

$$
(x y z) \text {, }
$$

$$
l^{2}=x_{0}^{2}+y_{0}^{2}+z_{0}^{2}
$$$$
4 A_{1} B_{1}=-1 \text {, }
$$

$$
\begin{aligned}
e b & =4 x_{0}^{\prime} A_{1}, \\
3 v & =4\left(1+B_{1}\right), \\
3 e_{1} & =4 z_{0}^{\prime}\left(A_{1} b^{-1}-a^{-1}\right), \\
e_{2} & =e_{1}+a^{-1} z_{0}^{\prime}, \\
v_{2} & =v-A_{1}^{-1} .
\end{aligned}
$$

The symbols like $\mathrm{ABC}$ are abbreviated equations.

\section{Construction of Periodic Solutions with Zeros Basic Amplitudes}

In this section, we use the suggested method for constructing the aimed solutions for the autonomous system (2). Consider the condition [4]

$$
p_{2}(0,0)=\dot{p}_{2}(0,0)=\dot{\gamma}_{2}(0, \varepsilon)=0 \text {. }
$$

The generating system for $(2)$ is obtained when $\varepsilon \longrightarrow \infty$ as follows:

$$
\begin{gathered}
4 \ddot{p}_{2}^{(0)}+p_{2}^{(0)}=0, \\
\ddot{\gamma}_{2}^{(0)}+\gamma_{2}^{(0)}=0 .
\end{gathered}
$$

The solutions for system (10) with a period $T_{0}=4 \pi$ are

$$
\begin{aligned}
& p_{2}^{(0)}=a_{0}^{*} \cos (0.5 \tau), \\
& \gamma_{2}^{(0)}=b_{0}^{*} \cos \tau,
\end{aligned}
$$

where $a_{0}^{*}$ and $b_{0}^{*}$ are constants. 
Let system (2) has periodic solutions with a period $T_{0}+\alpha$ in the form [5]

$$
\begin{aligned}
& p_{2}=a^{*} \cos \psi+\sum_{n=1}^{N} \varepsilon^{-n} p_{n}^{*}\left(a^{*}, \psi\right)+O\left(\varepsilon^{-N-1}\right), \\
& \gamma_{2}=b^{*} \cos \phi+\sum_{n=1}^{N} \varepsilon^{-n} \gamma_{n}^{*}\left(a^{*}, \phi\right)+O\left(\varepsilon^{-N-1}\right) .
\end{aligned}
$$

For these solutions, we let the initial conditions

$$
\begin{aligned}
& p_{2}(0, \varepsilon)=a^{*}=a_{0}^{*}+a^{*}(\varepsilon), \\
& \gamma_{2}(0, \varepsilon)=b^{*}=b_{0}^{*}+b^{*}(\varepsilon), \\
& \dot{\gamma}_{2}(0, \varepsilon)=0 .
\end{aligned}
$$

Here, $a^{*}(\varepsilon), b^{*}(\varepsilon) \longrightarrow 0$ at $\varepsilon \longrightarrow \infty$. Considering first integral (3) with conditions (13), we get

$$
\begin{aligned}
& 0<b_{0}^{*}=\left(1-\gamma_{0}^{\prime \prime 2}\right)^{1 / 2}\left(\gamma_{0}^{\prime \prime}\right)^{-1}<\infty, \\
& b^{*}(\varepsilon)=-\varepsilon^{-1} \nu\left[a_{0}^{*}+a^{*}(\varepsilon)\right]+\cdots .
\end{aligned}
$$

Let $a^{*}, \psi$, and $\phi$ are changed with time according to

$$
\dot{a}^{*}=\sum_{n=1}^{N} \varepsilon^{-n} A_{n}^{*}\left(a^{*}\right)+O\left(\varepsilon^{-N-1}\right),
$$

$$
\begin{aligned}
& \dot{\psi}=0.5+\sum_{n=1}^{N} \varepsilon^{-n} \psi_{n}\left(a^{*}\right)+O\left(\varepsilon^{-N-1}\right), \\
& \dot{\phi}=1+\sum_{n=1}^{N} \varepsilon^{-n} \phi_{n}\left(a^{*}\right)+O\left(\varepsilon^{-N-1}\right) .
\end{aligned}
$$

The following derivatives are obtained:

$$
\begin{aligned}
\dot{p}_{2}= & -0.5 a^{*} \sin \psi+O\left(\varepsilon^{-1}\right), \\
\dot{\gamma}_{2}= & -b^{*} \sin \phi+O\left(\varepsilon^{-1}\right), \\
\ddot{p}_{2}= & -0.25 a^{*} \cos \psi+\varepsilon^{-1}\left[0.25 \frac{\partial^{2} p_{1}^{*}}{\partial \psi^{2}}-a^{*} \psi_{1} \cos \psi-A_{1}^{*} \sin \psi\right] \\
& +\varepsilon^{-2}\left[A_{1}^{*} \frac{\partial^{2} p_{1}^{*}}{\partial a^{*} \partial \psi}-\left(A_{2}^{*}+2 A_{1}^{*} \psi_{1}\right) \sin \psi+A_{1}^{*} \frac{\mathrm{d} A_{1}^{*}}{\mathrm{~d} a^{*}} \cos \psi+0.25 \frac{\partial^{2} p_{2}^{*}}{\partial \psi^{2}}+\psi_{1} \frac{\partial^{2} p_{1}^{*}}{\partial \psi^{2}}-a^{*}\left(\psi_{1}^{2}+2 \psi_{2}\right) \cos \psi-a^{*} A_{1}^{*} \sin \psi \frac{\mathrm{d} \psi_{1}}{\mathrm{~d} a^{*}}\right]+O\left(\varepsilon^{-3}\right), \\
\ddot{\gamma}_{2}= & -b^{*} \cos \phi+\varepsilon^{-1}\left[\frac{\partial^{2} \gamma_{1}^{*}}{\partial \phi^{2}}-2 b^{*} \phi_{1} \cos \phi\right] \\
& +\varepsilon^{-2}\left[\frac{\partial^{2} \gamma_{2}^{*}}{\partial \phi^{2}}+2 \phi_{1} \frac{\partial^{2} \gamma_{1}^{*}}{\partial \phi^{2}}-b^{*}\left(\phi_{1}^{2}+2 \phi_{2}\right) \cos \phi+2 A_{1}^{*} \frac{\partial^{2} \gamma_{1}^{*}}{\partial a^{*} \partial \phi}-b^{*} A_{1}^{*} \frac{\mathrm{d} \phi_{1}}{\mathrm{~d} a^{*}} \sin \phi\right]+O\left(\varepsilon^{-3}\right) .
\end{aligned}
$$

Using equations (7), (12), and (18), we get

$$
\begin{aligned}
s_{11}^{(0)}= & a a_{0}^{* 2}\left(\cos ^{2} \psi_{0}-\cos ^{2} \psi\right)-0.25 b A_{1}^{-2} a_{0}^{* 2} \sin ^{2} \psi \\
& -2 b_{0}^{*}\left[x_{0}^{\prime}\left(\cos \phi_{0}-\cos \phi\right)+y_{0}^{\prime} \sin \phi\right], \\
s_{21}^{(0)}= & a_{0}^{*} b_{0}^{*}\left[a\left(\cos \psi_{0} \cos \phi_{0}-\cos \psi \cos \phi\right)+0.5 b A_{1}^{-1} \sin \psi \sin \phi\right], \\
s_{22}^{(0)}= & a\left[v a_{0}^{* 2}\left(\cos ^{2} \psi_{0}-\cos ^{2} \psi\right)+e b_{0}^{*}\left(\cos \phi_{0}-\cos \phi\right)+e_{1} b_{0}^{* 2}\left(\cos ^{2} \phi_{0}-\cos ^{2} \phi\right)\right] \\
& +b A_{1}^{-1}\left[0.25 v_{2} a_{0}^{* 2} \sin ^{2} \psi+a^{-1} y_{0}^{\prime} b_{0}^{*} \sin \phi+e_{2} b_{0}^{* 2} \sin ^{2} \phi\right],
\end{aligned}
$$

where $\psi_{0}$ and $\phi_{0}$ are the initial values of the corresponding functions.
Using (4), (12), (18), and (19), we obtain 


$$
\begin{aligned}
F^{(0)}= & 0.25 C_{1} A_{1}^{-1} a_{0}^{* 3} \cos \psi \sin ^{2} \psi+0.5 a_{0}^{*} b_{0}^{*} x_{0}^{\prime} \sin \psi \sin \phi \\
& +a^{-1} a_{0}^{*} b_{0}^{*} y_{0}^{\prime} \cos \psi \sin \phi+0.5 A_{1}^{-1}\left(A_{1}+a^{-1}\right) a_{0}^{*} b_{0}^{*} y_{0}^{\prime} \sin \psi \cos \phi \\
& -z_{0}^{\prime} a^{-1} a_{0}^{*} \cos \psi-0.75 v e_{1} a_{0}^{*} \cos \psi \\
& -0.25 a_{0}^{*} \cos \psi\left\{a a_{0}^{* 2}\left(\cos ^{2} \psi_{0}-\cos ^{2} \psi\right)-0.25 b A_{1}^{-2} a_{0}^{* 2} \sin ^{2} \psi-2 b_{0}^{*}\left[x_{0}^{\prime}\left(\cos \phi_{0}-\cos \phi\right)+y_{0}^{\prime} \sin \phi\right]\right\} \\
& +A_{1} b^{-1} x_{0}^{\prime} a_{0}^{*} b_{0}^{*}\left[a\left(\cos \psi_{0} \cos \phi_{0}-\cos \psi \cos \phi\right)+0.5 b A_{1}^{-1} \sin \psi \sin \phi\right] \\
\Phi^{(0)}= & 0.25\left(C_{1}-1\right) A_{1}^{-1} a_{0}^{* 2} b_{0}^{*} \sin 2 \psi \sin \phi+0.5 x_{0}^{\prime} b_{0}^{* 2}(1-\cos 2 \phi) \\
& +0.5 y_{0}^{\prime} b_{0}^{* 2} \sin 2 \phi-z_{0}^{\prime} b^{-1} b_{0}^{*} \cos \phi+x_{0}^{\prime} b^{-1} \\
& -0.125 A_{1}^{-2} a_{0}^{* 2} b_{0}^{*}(1-\cos 2 \psi) \cos \phi+0.75 v e+0.75 v e_{1} b_{0}^{*} \cos \phi \\
& -a a_{0}^{* 2} b_{0}^{*} \cos \psi_{0} \cos \phi+0.5 a a_{0}^{* 2} b_{0}^{*}(1+\cos 2 \psi) \cos \phi \\
& +0.125 b A_{1}^{-2} a_{0}^{* 2} b_{0}^{*}(1-\cos 2 \psi) \cos \phi+2 x_{0}^{\prime} b_{0}^{* 2} \cos \phi_{0} \cos \phi \\
& -x_{0}^{\prime} b_{0}^{* 2}(1+\cos 2 \phi)+y_{0}^{\prime} b_{0}^{* 2} \sin 2 \phi \\
& +a_{0}^{* 2} b_{0}^{*}\left(1+B_{1}\right)\left[0.5 b A_{1}^{-1} \sin \psi \sin \phi+a\left(\cos \psi_{0} \cos \phi_{0}-\cos \psi \cos \phi\right)\right] \cos \psi
\end{aligned}
$$

Substituting from (12), (18), and (20) into (2) and equating coefficients of $\varepsilon^{-1}$ in both sides, we get

$\frac{\partial^{2} p_{1}^{*}}{\partial \psi^{2}}+p_{1}^{*}=4 a_{0}^{*} \psi_{1} \cos \psi+4 A_{1}^{*} \sin \psi$

$\frac{\partial^{2} \gamma_{1}^{*}}{\partial \phi^{2}}+\gamma_{1}^{*}=2 b_{0}^{*} \phi_{1} \cos \phi$

$\frac{\partial^{2} p_{2}^{*}}{\partial \psi^{2}}+p_{2}^{*}=4 A_{2}^{*} \sin \psi+a_{0}^{*}\left[4 \psi_{2}+0.25 C_{1} A_{1}^{-1} a_{0}^{* 2}-3.25 a a_{0}^{* 2}-4 z_{0}^{\prime} a^{-1}-3 v e_{1}+0.125 b A_{1}^{-2} a_{0}^{* 2}+2 x_{0}^{\prime} b_{0}^{*} \cos \phi_{0}\right] \cos \psi$ $+0.25 a_{0}^{* 3}\left(a-C_{1} A_{1}^{-1}-0.25 b A_{1}^{-2}\right) \cos 3 \psi+4 a a_{0}^{*} x_{0}^{\prime} A_{1} b^{-1} b_{0}^{*} \cos \psi_{0} \cos \phi_{0}$

$+x_{0}^{\prime} a_{0}^{*} b_{0}^{*}\left(1-2 a A_{1} b^{-1}\right) \cos (\phi-\psi)-x_{0}^{\prime} a_{0}^{*} b_{0}^{*}\left(3+2 a A_{1} b^{-1}\right) \cos (\phi+\psi)$

$$
+y_{0}^{\prime} a_{0}^{*} b_{0}^{*}\left(2 a^{-1}-A_{1}^{-1} a^{-1}\right) \sin (\phi-\psi)+y_{0}^{\prime} a_{0}^{*} b_{0}^{*}\left(2+2 a^{-1}+A_{1}^{-1} a^{-1}\right) \sin (\phi+\psi),
$$

$\frac{\partial^{2} \gamma_{2}^{*}}{\partial \phi^{2}}+\gamma_{2}^{*}=\left[2 \phi_{2}-z_{0}^{\prime} b^{-1}+0.125 A_{1}^{-2} a_{0}^{* 2}(b-1)+0.75 v e_{1}-a a_{0}^{* 2} \cos ^{2} \psi_{0}-0.5 a B_{1} a_{0}^{* 2}+2 x_{0}^{\prime} b_{0}^{*} \cos \phi_{0}\right] b_{0}^{*} \cos \phi$

$$
\begin{aligned}
& -0.5 x_{0}^{\prime} b_{0}^{* 2}+x_{0}^{\prime} b^{-1}+0.75 v e+\left(1+B_{1}\right) a a_{0}^{* 2} b_{0}^{*} \cos \psi_{0} \cos \phi_{0} \cos \psi-0.67 x_{0}^{\prime} b_{0}^{* 2} \cos 2 \phi+1.5 y_{0}^{\prime} b_{0}^{* 2} \sin 2 \phi \\
& +0.5 a_{0}^{* 2}\left\{\left[0.25 A_{1}^{-2}(1-b)-a B_{1}+A_{1}^{-1} b_{0}^{*}(b-1)\right] \cos (2 \psi-\phi)\right. \\
& \left.+\left[0.25 A_{1}^{-2}(1-b)-a B_{1}-A_{1}^{-1} b_{0}^{*}(b-1)\right] \cos (2 \psi+\phi)\right\} .
\end{aligned}
$$

Canceling singular terms from (21) as in [6], we get 


$$
\begin{aligned}
& \psi_{1}=A_{1}^{*}=\phi_{1}=A_{2}^{*}=0, \\
& \psi_{2}=\left[-0.06 C_{1} A_{1}^{-1} a_{0}^{* 2}+0.81 a a_{0}^{* 2}+z_{0}^{\prime} a^{-1}+0.75 v e_{1}-0.02 b A_{1}^{-2} a_{0}^{* 2}-0.5 x_{0}^{\prime} b_{0}^{*} \cos \phi_{0}\right], \\
& \phi_{2}=0.5\left[z_{0}^{\prime} b^{-1}-0.125 A_{1}^{-2} a_{0}^{* 2}(b-1)-0.75 v e_{1}+a a_{0}^{* 2}\left(0.5 B_{1}+\cos ^{2} \psi_{0}\right)-2 x_{0}^{\prime} b_{0}^{*} \cos \phi_{0}\right] .
\end{aligned}
$$
obtain

Substituting from (22) into (15)-(17) and integrating, we

$$
\begin{aligned}
a^{*} & =a_{0}^{*} \text { (arbitrary const.) } \\
\psi & =0.5 \tau+0.5 \varepsilon^{-2}\left[-0.125 C_{1} A_{1}^{-1} a_{0}^{* 2}-0.375 a a_{0}^{* 2}+2 a a_{0}^{* 2}+2 z_{0}^{\prime} a^{-1}+1.5 v e_{1}-0.31 b A_{1}^{-2} a_{0}^{* 2}-x_{0}^{\prime} b_{0}^{*} \cos \phi_{0}\right] \tau \\
\phi & =\tau+0.5 \varepsilon^{-2}\left[z_{0}^{\prime} b^{-1}-0.125 A_{1}^{-2} a_{0}^{* 2}(b-1)-0.75 v e_{1}+a a_{0}^{* 2}\left(1+0.5 B_{1}\right)-2 x_{0}^{\prime} b_{0}^{*}\right] \tau
\end{aligned}
$$

From the previous results, we get

$$
\begin{aligned}
& \psi(0)=\psi_{0}=0, \\
& \phi(0)=\phi_{0}=0 .
\end{aligned}
$$

From (13) and (23), we obtain $a^{*}$ from the order greater than $O\left(\varepsilon^{-2}\right)$.

The periodic solutions $p_{2}$ and $\gamma_{2}$ are obtained by substituting (22) and (23) into (21) and using (12) and (14). Finally, the periodic solutions $p_{1}, q_{1}, r_{1}, \gamma_{1}, \gamma_{1}^{\prime}$, and $\gamma_{1}^{\prime \prime}$ are obtained from (5), (19), (23), and (24).

\section{Construction of Periodic Solutions with Nonzeros Basic Amplitudes}

We use the large parameter method [7] for constructing the periodic solutions with nonzeros basic amplitudes for system (2) when $\mathrm{A}<\mathrm{B}<\mathrm{C}$ or $\mathrm{A}>\mathrm{B}>\mathrm{C}$. Consider generating system (10) has periodic solutions with a period $T_{0}=2 \pi n$ as follows:

$$
\begin{aligned}
& p_{2}^{(0)}(\tau)=E \cos (0.5 \tau-\mu) \\
& \gamma_{2}^{(0)}(\tau)=M_{3} \cos \tau
\end{aligned}
$$

where $E=\sqrt{M_{1}^{2}+M_{2}^{2}}, \mu=\tan ^{-1}\left(M_{2} / M_{1}\right)$, and $M_{1}, M_{2}$, and $M_{3}$ are constants.

Let system (2) has periodic solutions with a period $T_{0}+\alpha$ that reduces to generating solutions $(21)$ when $\varepsilon \longrightarrow \infty$, where $\alpha$ is a function of $\varepsilon$ such that $\alpha(\infty)=0$. Consider the following initial conditions:

$$
\begin{aligned}
& p_{2}(0, \varepsilon)=\tilde{M}_{1}, \\
& \dot{p}_{2}(0, \varepsilon)=00.5 \tilde{M}_{2}, \\
& \gamma_{2}(0, \varepsilon)=\tilde{M}_{3}, \\
& \dot{\gamma}_{2}(0, \varepsilon)=0 .
\end{aligned}
$$

The notation $\sim$ denotes the following substitution:

$$
M_{i} \longrightarrow \tilde{M}_{i}=M_{i}+\beta_{i}, \quad i=1,2,3,
$$

where $\beta_{1}, 0.5 \beta_{2}$, and $\beta_{3}$ represent the deviations of the initial values of the required solutions from their values of the generating ones $M_{1}, M_{2}$, and $M_{3}$, respectively. These deviations are functions of $\varepsilon$ and vanish when $\varepsilon \longrightarrow \infty$. Now, we construct the required solutions in the following forms [8]:

$$
\begin{aligned}
& p_{2}=\widetilde{E} \cos (\psi-\mu)+\sum_{n=1}^{N} \varepsilon^{-n} p_{n}^{*}(\widetilde{E}, \psi)+O\left(\varepsilon^{-N-1}\right), \\
& \gamma_{2}=\widetilde{M}_{3} \cos \phi+\sum_{n=1}^{N} \varepsilon^{-n} \gamma_{n}^{*}(\widetilde{E}, \phi)+O\left(\varepsilon^{-N-1}\right),
\end{aligned}
$$

where $p_{n}^{*}$ and $\gamma_{n}^{*}$ are periodic functions in $\psi$ and $\phi$, respectively. The quantity $\widetilde{M}_{3}$ is determined from the first integral (3). Let $\widetilde{E}, \psi$, and $\phi$ are changed with time according to

$$
\begin{aligned}
& \frac{\mathrm{d} \widetilde{E}}{\mathrm{~d} \tau}=\sum_{n=1}^{N} \varepsilon^{-n} E_{n}(\widetilde{E})+O\left(\varepsilon^{-N-1}\right), \\
& \frac{\mathrm{d} \psi}{\mathrm{d} \tau}=0.5+\sum_{n=1}^{N} \varepsilon^{-n} \psi_{n}(\widetilde{E})+O\left(\varepsilon^{-N-1}\right),
\end{aligned}
$$




$$
\frac{\mathrm{d} \phi}{\mathrm{d} \tau}=1+\sum_{n=1}^{N} \varepsilon^{-n} \phi_{n}(\widetilde{E})+O\left(\varepsilon^{-N-1}\right) .
$$

Substituting initial conditions (26) into integral (3), when $\tau=0$, we deduce that

$$
0<M_{3}=\frac{\sqrt{1-\gamma_{0}^{\prime \prime 2}}}{\gamma_{0}^{\prime \prime}}<\infty,
$$

$$
\beta_{3}=-\varepsilon^{-1} \nu \tilde{M}_{1}+\cdots
$$

The derivatives become

$$
\begin{aligned}
& \dot{p}_{2}=\frac{\mathrm{d} \widetilde{E}}{\mathrm{~d} \tau} \frac{\partial p_{2}}{\partial \widetilde{E}}+\frac{\mathrm{d} \psi}{\mathrm{d} \tau} \frac{\partial p_{2}}{\partial \psi} \\
& \dot{\gamma}_{2}=\frac{\mathrm{d} \widetilde{E}}{\mathrm{~d} \tau} \frac{\partial \gamma_{2}}{\partial \widetilde{E}}+\frac{\mathrm{d} \phi}{\mathrm{d} \tau} \frac{\partial \gamma_{2}}{\partial \phi} \\
& \ddot{p}_{2}=\left(\frac{\mathrm{d} \widetilde{E}}{\mathrm{~d} \tau}\right)^{2} \frac{\partial^{2} p_{2}}{\partial \widetilde{E}^{2}}+\frac{\mathrm{d}^{2} \widetilde{E}}{\mathrm{~d} \tau^{2}} \frac{\partial p_{2}}{\partial \widetilde{E}}+2 \frac{\mathrm{d} \widetilde{E}}{\mathrm{~d} \tau} \frac{\mathrm{d} \psi}{\mathrm{d} \tau} \frac{\partial^{2} p_{2}}{\partial \widetilde{E} \partial \psi}+\left(\frac{\mathrm{d} \psi}{\mathrm{d} \tau}\right)^{2} \frac{\partial^{2} p_{2}}{\partial \psi^{2}}+\frac{\mathrm{d}^{2} \psi}{\mathrm{d} \tau^{2}} \frac{\partial p_{2}}{\partial \psi} \\
& \ddot{\gamma}_{2}=\left(\frac{\mathrm{d} \widetilde{E}}{\mathrm{~d} \tau}\right)^{2} \frac{\partial^{2} \gamma_{2}}{\partial \widetilde{E}^{2}}+\frac{\mathrm{d}^{2} \widetilde{E}}{\mathrm{~d} \tau^{2}} \frac{\partial \gamma_{2}}{\partial \widetilde{E}}+2 \frac{\mathrm{d} \widetilde{E}}{\mathrm{~d} \tau} \frac{\mathrm{d} \phi}{\mathrm{d} \tau} \frac{\partial^{2} \gamma_{2}}{\partial \widetilde{E} \partial \phi}+\left(\frac{\mathrm{d} \phi}{\mathrm{d} \tau}\right)^{2} \frac{\partial^{2} \gamma_{2}}{\partial \phi^{2}}+\frac{\mathrm{d}^{2} \phi}{\mathrm{d} \tau^{2}} \frac{\partial \gamma_{2}}{\partial \phi}
\end{aligned}
$$

Using equations (7), (28), and (33), we get

$$
\begin{aligned}
s_{11}^{(0)}= & E^{2}\left[\left(a \cos ^{2} \mu-0.5\right)+0.25 b A_{1}^{-2}\left(\sin ^{2} \mu-0.5\right)+0.5\left(0.25 b A_{1}^{-2}-a\right) \cos (\tau-2 \mu)\right]-2 M_{3}\left[x_{0}^{\prime}(1-\cos \tau)+y_{0}^{\prime} \sin \tau\right], \\
s_{21}^{(0)}= & M_{3} E\left[a \cos \mu+0.5\left(0.5 b A_{1}^{-1}-a\right) \cos (0.5 \tau+\mu)-0.5\left(0.5 b A_{1}^{-1}+a\right) \cos (1.5 \tau-\mu)\right], \\
s_{22}^{(0)}= & E^{2}\left[\nu a\left(\cos ^{2} \mu-0.5\right)-0.25 b A_{1}^{-1} \nu_{2}\left(\sin ^{2} \mu-0.5\right)-0.5\left(\nu a+0.25 b A_{1}^{-1} v_{2}\right) \cos (\tau-2 \mu)\right] \\
& +0.5 M_{3}^{2}\left(e_{1} a+b A_{1}^{-1} e_{2}\right)(1-\cos 2 \tau)+M_{3}\left[a e(1-\cos \tau)+b y_{0}^{\prime} a^{-1} A_{1}^{-1} \sin \tau\right] .
\end{aligned}
$$

Using (4), (28), (33), and (34), we obtain

$$
\begin{aligned}
F^{(0)}= & 0.25 C_{1} A_{1}^{-1} E^{3} \cos (0.5 \tau-\mu) \sin ^{2}(0.5 \tau-\mu)+E M_{3} \sin \tau\left[0.5 x_{0}^{\prime} \sin (0.5 \tau-\mu)+y_{0}^{\prime} a^{-1} \cos (0.5 \tau-\mu)\right] \\
& +0.5 y_{0}^{\prime} A_{1}^{-1}\left(A_{1}+a^{-1}\right) M_{3} E \cos \tau \sin (0.5 \tau-\mu)-E\left(z_{0}^{\prime} a^{-1}+0.75 v e_{1}\right) \cos (0.5 \tau-\mu) \\
& -0.25 E \cos (0.5 \tau-\mu)\left\{E^{2}\left[a \cos ^{2} \mu-0.5+0.25 b A_{1}^{-2}\left(\sin ^{2} \mu-0.5\right)+0.5\left(0.25 b A_{1}^{-2}-a\right) \cos (\tau-2 \mu)\right]\right. \\
& \left.-2 M_{3}\left[x_{0}^{\prime}(1-\cos \tau)+y_{0}^{\prime} \sin \tau\right]\right\} \\
& +A_{1} b^{-1} x_{0}^{\prime} M_{3} E\left[a \cos \mu+0.5\left(0.5 b A_{1}^{-1}-a\right) \cos (0.5 \tau+\mu)-0.5\left(0.5 b A_{1}^{-1}+a\right) \cos (1.5 \tau-\mu)\right], \\
\Phi^{(0)}= & b^{-1} x_{0}^{\prime}-0.5 M_{3}^{2} x_{0}^{\prime}+0.75 v e-\left\{z_{0}^{\prime} b^{-1} M_{3}+0.125 A_{1}^{-2} M_{3} E^{2}-0.75 v e_{1} M_{3}\right. \\
& \left.+M_{3} E^{2}\left[a\left(\cos ^{2} \mu-0.5\right)+0.25 b A_{1}^{-2}\left(\sin ^{2} \mu-0.5\right)\right]-2 M_{3}^{2} x_{0}^{\prime}+0.5 a\left(1+B_{1}\right) M_{3} E^{2}\right\} \cos \tau \\
& -1.5 M_{3}^{2}\left(x_{0}^{\prime} \cos 2 \tau-y_{0}^{\prime} \sin 2 \tau\right)+\left(1+B_{1}\right) M_{3} E^{2} a \cos \mu(\cos 0.5 \tau \cos \mu+\sin 0.5 \tau \sin \mu) \\
& +0.25 E^{2} M_{3}\left[0.5\left(1-C_{1}\right) A_{1}^{-1}+0.25 A_{1}^{-2}(1-b)+a-\left(1+B_{1}\right)\left(0.5 A_{1}^{-1}+a\right)\right] \cos 2(\mu-\tau) \\
& +0.25 M_{3} E^{2} \times\left[0.5 A_{1}^{-1}\left(C_{1}-1\right)+0.25 A_{1}^{-2}(1-b)+a+\left(1+B_{1}\right)\left(0.5 b A_{1}^{-1}-a\right)\right] \cos 2 \mu .
\end{aligned}
$$


Substituting from (28), (33), and (35) into initial system (2) and equating coefficients of $\varepsilon^{-1}$ and $\varepsilon^{-2}$ in both sides, we obtain the following:

Coefficients of $\varepsilon^{-1}$ :

$$
\begin{aligned}
\frac{\partial^{2} p_{1}^{*}}{\partial \psi^{2}}+p_{1}^{*}= & 4\left(E \psi_{1} \cos \mu-E_{1} \sin \mu\right) \cos \psi \\
& +4\left(E \psi_{1} \sin \mu+E_{1} \cos \mu\right) \sin \psi, \\
\frac{\partial^{2} \gamma_{1}^{*}}{\partial \phi^{2}}+\gamma_{1}^{*}= & 2 \phi_{1} M_{3} \cos \phi .
\end{aligned}
$$

We neglect the singular terms [4] to get

$$
\begin{aligned}
E \psi_{1} \cos \mu-E_{1} \sin \mu & =0, \\
E \psi_{1} \sin \mu+E_{1} \cos \mu & =0, \\
\phi_{1} & =0,
\end{aligned}
$$

such that determinant (37) becomes

$$
\Delta=E\left|\begin{array}{cc}
\cos \mu & -\sin \mu \\
\sin \mu & \cos \mu
\end{array}\right|=E\left(\cos ^{2} \mu+\sin ^{2} \mu\right)=E \neq 0 .
$$

For this case, the solution of (37) becomes

$$
\psi_{1}=E_{1}=0 .
$$

The particular solutions for (36) become

$$
p_{1}^{*}=\gamma_{1}^{*}=0 .
$$

Coefficients of $\varepsilon^{-2}$ :

$\frac{\mathrm{d}^{2} p_{2}^{*}}{\mathrm{~d} \tau^{2}}+0.25 p_{2}^{*}=\left[E_{2} \cos \mu+E \psi_{2} \sin \mu\right] \sin 0.5 \tau$

$$
\begin{aligned}
& -\left\{E_{2} \sin \mu-\left[E \psi_{2}+0.06 C_{1} A_{1}^{-1} E^{3}-z_{0}^{\prime} a^{-1} E-0.75 v e_{1} E-0.25 E^{3} a\left(\cos ^{2} \mu-0.5\right)-0.06 E^{3} b A_{1}^{-2}\left(\sin ^{2} \mu-0.5\right)\right.\right. \\
& \left.\left.-0.06 E^{3}\left(0.25 b A_{1}^{-2}-a\right)+0.5 M_{3} x_{0}^{\prime} E\right] \cos \mu\right\} \cos 0.5 \tau \\
& +A_{1} b^{-1} x_{0}^{\prime} M_{3} E a \cos \mu-0.06 E^{3}\left(C_{1} A_{1}^{-1}+0.25 b A_{1}^{-2}-a\right)(\cos 3 \mu \cos 1.5 \tau+\sin 3 \mu \sin 1.5 \tau) \\
& +M_{3} E\left\{\left[0.5 A_{1} b^{-1}\left(0.5 b A_{1}^{-1}-a\right)\right] x_{0}^{\prime} \cos \mu+0.5\left[a^{-1}-0.5 A_{1}^{-1}\left(A_{1}+a^{-1}\right)+0.5\right] y_{0}^{\prime} \sin \mu\right\} \cos 0.5 \tau \\
& -0.5 M_{3} E\left\{\left[A_{1} b^{-1}\left(0.5 b A_{1}^{-1}-a\right)\right] x_{0}^{\prime} \sin \mu-\left[a^{-1}-\left(1+A_{1}^{-1} a^{-1}\right)+0.5\right] y_{0}^{\prime} \cos \mu\right\} \sin 0.5 \tau \\
& -0.5 M_{3} E\left\{\left[0.25+\left(0.5+A_{1} b^{-1} a\right)\right] x_{0}^{\prime} \cos \mu+\left[a^{-1}+\left(1+A_{1}^{-1} a^{-1}\right)+0.5\right] y_{0}^{\prime} \sin \mu\right\} \cos 1.5 \tau \\
& -0.5 M_{3} E\left\{1.5 A_{1} b^{-1} a x_{0}^{\prime} \sin \mu-\left[a^{-1}+\left(1+A_{1}^{-1} a^{-1}\right)+0.5\right] y_{0}^{\prime} \cos \mu\right\} \sin 1.5 \tau
\end{aligned}
$$

$$
\begin{aligned}
\frac{\mathrm{d}^{2} \gamma_{2}^{*}}{\mathrm{~d} \tau^{2}}+\gamma_{2}^{*}= & x_{0}^{\prime}\left(b^{-1}-0.5 M_{3}^{2}\right)+0.75 v e \\
& +M_{3}\left\{2 \phi_{2}-z_{0}^{\prime} b^{-1}-0.125 A_{1}^{-2} E^{2}+0.75 v e_{1}-E^{2}\left[a\left(\cos ^{2} \mu-0.5\right)+0.25 b A_{1}^{-2}\left(\sin ^{2} \mu-0.5\right)\right]\right. \\
& \left.+2 M_{3} x_{0}^{\prime}-0.5 a E^{2}\left(1+B_{1}\right)\right\} \cos \tau \\
& +1.5 M_{3}^{2}\left(y_{0}^{\prime} \sin 2 \tau-x_{0}^{\prime} \cos 2 \tau\right)+\left(1+B_{1}\right) M_{3} E^{2} a(\cos \mu \cos 0.5 \tau+\sin \mu \sin 0.5 \tau) \cos \mu \\
& +0.125 M_{3} E^{2}\left[\left(1-C_{1}\right) A_{1}^{-1}+0.5 A_{1}^{-2}-\left(0.5 b A_{1}^{-2}-2 a\right)-\left(1+B_{1}\right)\left(b A_{1}^{-1}+2 a\right)\right](\cos 2 \mu \cos 2 \tau+\sin 2 \mu \sin 2 \tau) \\
& +0.125 M_{3} E^{2}\left[\left(C_{1}-1\right) A_{1}^{-1}+0.5 A_{1}^{-2}-\left(0.5 b A_{1}^{-2}-2 a\right)+\left(1+B_{1}\right)\left(b A_{1}^{-1}-2 a\right)\right] \cos 2 \mu
\end{aligned}
$$

Neglecting singular terms from (42) and (43) yields [4]

$$
\begin{aligned}
& E_{2}=0.125 E \sin 2 \mu\left[0.25 C_{1} A_{1}^{-1} E^{2}-4 z_{0}^{\prime} a^{-1}-3 v e_{1}-E^{2} a\left(\cos ^{2} \mu-0.5\right)-0.25 E^{2} b A_{1}^{-2}\left(\sin ^{2} \mu-0.5\right)-0.25 E^{2}\left(0.25 b A_{1}^{-2}-a\right)+2 M_{3} x_{0}^{\prime}\right], \\
& \psi_{2}=0.25 \cos ^{2} \mu\left[-0.25 C_{1} A_{1}^{-1} E^{2}+4 z_{0}^{\prime} a^{-1}+3 v e_{1}+E^{2} a\left(\cos ^{2} \mu-0.5\right)+0.25 b E^{2} A_{1}^{-2}\left(\sin ^{2} \mu-0.5\right)+0.25 E^{2}\left(0.25 b A_{1}^{-2}-a\right)-2 M_{3} x_{0}^{\prime}\right], \\
& \phi_{2}=0.5\left\{z_{0}^{\prime} b^{-1}+0.125 A_{1}^{-2} E^{2}-0.75 v e_{1}+E^{2}\left[a\left(\cos ^{2} \mu-0.5\right)+0.25 b A_{1}^{-2}\left(\sin ^{2} \mu-0.5\right)\right]-2 M_{3} x_{0}^{\prime}+0.5 a\left(1+B_{1}\right) E^{2}\right\} .
\end{aligned}
$$


Substituting from (38), (40), and (44) into (29) and (30) and integrating, we get

$$
\begin{aligned}
2 \widetilde{E}= & 2 E-\varepsilon^{-2} E \sin 2 \mu\left[-0.25 C_{1} A_{1}^{-1} E^{2}+4 z_{0}^{\prime} a^{-1}+3 v e_{1}+E^{2} a\left(\cos ^{2} \mu-0.5\right)\right. \\
& \left.+0.25 b E^{2} A_{1}^{-2}\left(\sin ^{2} \mu-0.5\right)+0.25 E^{2}\left(0.25 b A_{1}^{-2}-a\right)-2 M_{3} x_{0}^{\prime}\right] \tau+\cdots, \\
2 \psi= & +0.5 \varepsilon^{-2}\left[-0.25 C_{1} A_{1}^{-1} E^{2}+4 z_{0}^{\prime} a^{-1}+3 v e_{1}+E^{2} a\left(\cos ^{2} \mu-0.5\right)\right. \\
& \left.+0.25 b \cdot E^{2} A_{1}^{-2}\left(\sin ^{2} \mu-0.5\right)+0.25 E^{2}\left(0.25 b A_{1}^{-2}-a\right)-2 M_{3} x_{0}^{\prime}\right] \cos ^{2} \mu \tau+\cdots, \\
\phi= & \tau+0.25 \varepsilon^{-2}\left\{2 z_{0}^{\prime} b^{-1}+0.25 A_{1}^{-2} E^{2}-1.5 v e_{1}+E^{2}\left[2 a\left(\cos ^{2} \mu-0.5\right)+0.5 b A_{1}^{-2}\left(\sin ^{2} \mu-0.5\right)\right]\right. \\
& \left.-4 M_{3} x_{0}^{\prime}+a\left(1+B_{1}\right) E^{2}\right\} \tau+\cdots .
\end{aligned}
$$

Substituting (44) into (42) and (43) and solving the resulted equations, we get $p_{2}^{*}$ and $\gamma_{2}^{*}$. The periodic solutions $p_{2}$ and $\gamma_{2}$ are constructed using (28), (32), (41), and (45). Using (5) and (34), we get the first terms of the required solutions as follows:

$$
\begin{aligned}
p_{1}= & M_{1} \cos 0.5 \tau+M_{2} \sin 0.5 \tau-\varepsilon^{-1}\left(\frac{x_{0}^{\prime}}{b B_{1}}-e_{1} M_{3} \cos \tau\right)+\cdots, \\
q_{1}= & 0.5 A_{1}^{-1}\left(M_{1} \sin 0.5 \tau-M_{2} \cos 0.5 \tau\right)+\varepsilon^{-1}\left(\frac{y_{0}^{\prime}}{a A_{1}}+e_{2} A_{1}^{-1} M_{3} \sin \tau\right)+\cdots, \\
r_{1}= & +0.25 \varepsilon^{-2}\left\{2 a M_{1}^{2}-E^{2}+0.5 b A_{1}^{-2}\left(M_{2}^{2}-0.5 E^{2}\right)+\left(0.25 b A_{1}^{-2}-a\right)\left[\left(M_{1}^{2}-M_{2}^{2}\right) \cos \tau+2 M_{1} M_{2} \sin \tau\right]\right. \\
& \left.-4 M_{3}\left[x_{0}^{\prime}(1-\cos \tau)+y_{0}^{\prime} \sin \tau\right]\right\}+\cdots, \\
\gamma_{1}= & M_{3} \cos \tau+\varepsilon^{-1} \nu\left(-M_{1} \cos \tau+M_{1} \cos 0.5 \tau+M_{2} \sin 0.5 \tau\right)+\cdots, \\
\gamma_{1}^{\prime}= & -M_{3} \sin \tau+\varepsilon^{-1}\left[\nu M_{1} \sin \tau+0.5 v_{2}\left(-M_{1} \sin 0.5 \tau+M_{2} \cos 0.5 \tau\right)\right]+\cdots, \\
\gamma_{1}^{\prime \prime}= & +\varepsilon^{-1} M_{3} E\left[a \cos \mu+0.5\left(b \omega A_{1}^{-1}-a\right) \cos ^{2}(0.5 \tau-\mu)-0.25\left(b A_{1}^{-1}+2 a\right) \cos (1.5 \tau-\mu)\right] \\
& +\varepsilon^{-2}\left\{M_{3}(1-a)^{-1} x_{0}^{\prime}+\frac{0.5 M_{3}^{2} z_{0}^{\prime}(a-b)}{(a+b-1)}+M_{3}(1-b)^{-1} y_{0}^{\prime} \sin \tau-M_{3}(1-a)^{-1} x_{0}^{\prime} \cos \tau-\frac{0.5 M_{3}^{2} z_{0}^{\prime}(a-b) \cos 2 \tau}{(a+b-1)}\right. \\
& +E^{2}\left[\nu a\left(\cos ^{2} \mu-0.5\right)-0.25 b A_{1}^{-1} v_{2}\left(\sin ^{2} \mu-0.5\right)-0.125\left(4 v a+b A_{1}^{-1} v_{2}\right) \cos 2(0.5 \tau-\mu)\right] \\
& \left.-0.5 E^{2}\left[\left(a \cos ^{2} \mu-0.5\right)+0.25 b A_{1}^{-2}\left(\sin ^{2} \mu-0.5\right)+0.125\left(b A_{1}^{-2}-4\right) \cos 2(0.5 \tau-\mu)\right]\right\}+\cdots
\end{aligned}
$$

The correction of the period is

$$
\alpha(\varepsilon)=\varepsilon^{-2} \pi n\left\{2 M_{3} x_{0}^{\prime}-2 z_{0}^{\prime}-0.125 A_{1}^{-2} E^{2}-E^{2}\left[a\left(\cos ^{2} \mu-0.5\right)+0.25 b A_{1}^{-2}\left(\sin ^{2} \mu-0.5\right)\right]-0.5 a E^{2}\left(1+B_{1}\right)\right\}+\cdots
$$

\section{Geometric Interpretation of Motion}

In this section, we describe the body motion using Euler's angles $\xi, \zeta$, and $\eta$ which come from the obtained solutions (Figure 2). Replacing the time $t$ by $t+t_{0}$ where $t_{0}$ is an arbitrary interval, the periodic solutions remain periodic since the initial system is autonomous [9]. For this case, we obtain from (32),

$$
\begin{aligned}
& \eta_{0}=0.5 \pi+r_{0}^{-1} t_{0}+\cdots, \\
& \xi_{0}=\tan ^{-1} M_{3},
\end{aligned}
$$

where $\eta_{0}$ are $\xi_{0}$ are arbitrary initial angles.

Making use of (46) and (49) when $\tau=r_{0}^{-1} t$, we find Euler's angles as follows: 


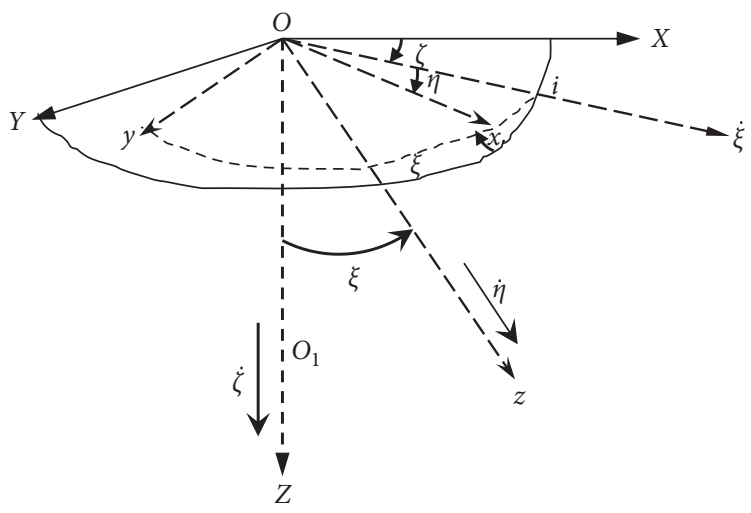

FIgURE 2: The rotational planes in terms of Euler's angles.

$$
\begin{aligned}
& \xi=\xi_{0}-\varepsilon^{-1} E\left[\xi_{1}\left(t+t_{0}\right)-\xi_{1}\left(t_{0}\right)\right]-\varepsilon^{-2}\left[\xi_{2}\left(t+t_{0}\right)-\xi_{2}\left(t_{0}\right)\right]+\cdots, \\
& \zeta=\zeta_{0}+0.5 M g \ell C^{-1} r_{0} \cos ^{2} \xi_{0} Q_{10} t+0.5 \varepsilon^{-1} \sec \xi_{0}\left[\zeta_{1}\left(t+t_{0}\right)-\zeta_{1}\left(t_{0}\right)\right]+0.5 \varepsilon^{-2} \cos \xi_{0}\left[\zeta_{2}\left(t+t_{0}\right)-\zeta_{2}\left(t_{0}\right)\right]+\cdots \\
& \eta=\eta_{0}+\left(r_{0}^{-1}-0.5 M g \ell C^{-1} r_{0} \cos ^{3} \xi_{0} h_{10}\right) t-0.5 \varepsilon^{-1} \cot \xi_{0}\left[\eta_{1}\left(t+t_{0}\right)-\eta_{1}\left(t_{0}\right)\right]-0.5 \varepsilon^{-2} \cos ^{2} \xi_{0}\left[\eta_{2}\left(t+t_{0}\right)-\eta_{2}\left(t_{0}\right)\right]+\cdots
\end{aligned}
$$

where

$$
\begin{aligned}
& \xi_{1}(t)=0.5\left(0.5 b A_{1}^{-1}-a\right) \cos \left(\frac{t}{2 r_{0}}+\mu\right)-0.5\left(0.5 b A_{1}^{-1}+a\right) \cos \left(\frac{3 t}{2 r_{0}}-\mu\right), \\
& \xi_{2}(t)=y_{0}^{\prime} a^{-1} A_{1}^{-1} \sin \frac{t}{r_{0}}+b^{-1} B_{1}^{-1} x_{0}^{\prime} \cos \frac{t}{r_{0}}-0.5 \tan \xi_{0} z_{0}^{\prime}\left(\frac{a-b}{a+b-1}\right) \cos 2 \frac{t}{r_{0}} \\
& -0.5 E^{2} \cot \xi_{0}\left[a(\nu-0.5)+0.25 b A_{1}^{-1}\left(\nu_{2}+0.5 A_{1}^{-1}\right)\right] \cos \left(\frac{t}{r_{0}}-2 \mu\right) \\
& \zeta_{1}(t)=\eta_{1}(t)=0.67\left(1+0.5 A_{1}^{-1}\right)\left(M_{1} \sin \frac{3 t}{2 r_{0}}-M_{2} \cos \frac{3 t}{2 r_{0}}\right)+\left(2-A_{1}^{-1}\right)\left(M_{2} \cos \frac{t}{2 r_{0}}+M_{1} \sin \frac{t}{2 r_{0}}\right) \text {, } \\
& \zeta_{2}(t)=\left(Q_{11}+Q_{13}+Q_{16}\right) \sin \frac{t}{r_{0}}-\left(Q_{11}^{\prime}+Q_{13}^{\prime}-Q_{16}^{\prime}\right) \cos \frac{t}{r_{0}} \\
& +0.5\left(Q_{12} \sin \frac{2 t}{r_{0}}-Q_{12}^{\prime} \cos \frac{2 t}{r_{0}}\right)+2\left(Q_{14} \sin \frac{t}{2 r_{0}}+Q_{14}^{\prime} \cos \frac{t}{2 r_{0}}\right)+0.67\left(Q_{15} \sin \frac{3 t}{2 r_{0}}-Q_{15}^{\prime} \cos \frac{3 t}{2 r_{0}}\right) \\
& \eta_{2}(t)=h_{11} \sin \frac{t}{r_{0}}-h_{11}^{\prime} \cos \frac{t}{r_{0}}+0.5\left(h_{12} \sin \frac{2 t}{r_{0}}-h_{12}^{\prime} \cos \frac{2 t}{r_{0}}\right)+\left(h_{13} \sin \frac{t}{r_{0}}-h_{13}^{\prime} \cos \frac{t}{r_{0}}\right)+2\left(h_{14} \sin \frac{t}{2 r_{0}}+h_{14}^{\prime} \cos \frac{t}{2 r_{0}}\right) \\
& +0.67\left(h_{15} \sin \frac{3 t}{2 r_{0}}-h_{15}^{\prime} \cos \frac{3 t}{2 r_{0}}\right)+\left(h_{16} \sin \frac{t}{r_{0}}+h_{16}^{\prime} \cos \frac{t}{r_{0}}\right)+0.34\left(h_{17} \sin \frac{3 t}{r_{0}}-h_{17}^{\prime} \cos \frac{3 t}{r_{0}}\right) .
\end{aligned}
$$


TABLE 1: The analytical solutions $p_{2}, \gamma_{2}$, and their derivatives.

\begin{tabular}{|c|c|c|c|c|}
\hline$t$ & $p_{2}$ & $\gamma_{2}$ & $\dot{p}_{2}$ & $\dot{\gamma}_{2}$ \\
\hline 0 & 1.5 & 11.06602 & 0.8164966 & $8.60977 E-05$ \\
\hline 10 & 2.018443 & 8.279188 & 0.6022027 & -7.342405 \\
\hline 20 & 2.361099 & 1.322297 & 0.3354627 & -10.98657 \\
\hline 30 & 2.498126 & -6.300518 & 0.03950729 & -9.096887 \\
\hline 40 & 2.417591 & -10.74971 & -0.259889 & -2.625185 \\
\hline 50 & 2.126507 & -9.784271 & -0.5366514 & 5.168785 \\
\hline 60 & 1.650225 & -3.890493 & -0.7666767 & 10.35926 \\
\hline 70 & 1.030224 & 3.962964 & -0.9299318 & 10.33185 \\
\hline 80 & 0.3205004 & 9.82036 & -1.012199 & 5.10033 \\
\hline 90 & -0.4171353 & 10.73134 & -1.006313 & -2.700216 \\
\hline 100 & -1.118443 & 6.237032 & -0.9127875 & -9.140733 \\
\hline 110 & -1.722344 & -1.398863 & -0.7397668 & -10.97719 \\
\hline 120 & -2.176246 & -8.330236 & -0.5023198 & -7.284575 \\
\hline 130 & -2.440619 & -11.06585 & -0.2211253 & 0.07719428 \\
\hline 140 & -2.492437 & -8.227805 & 0.07932674 & 7.400064 \\
\hline 150 & -2.327188 & -1.245598 & 0.3728705 & 10.9956 \\
\hline 160 & -1.959263 & 6.363904 & 0.6339409 & 9.052767 \\
\hline 170 & -1.420706 & 10.76787 & 0.839801 & 2.550172 \\
\hline 180 & -0.7584189 & 9.748108 & 0.9725226 & -5.236892 \\
\hline 190 & -0.0300812 & 3.818282 & 1.020547 & -10.38613 \\
\hline 200 & 0.7008771 & -4.034786 & 0.9796914 & -10.30393 \\
\hline 210 & 1.370795 & -9.855532 & 0.8535145 & -5.031701 \\
\hline 220 & 1.921331 & -10.71208 & 0.6530044 & 2.774997 \\
\hline 230 & 2.304537 & -6.172976 & 0.3956243 & 9.183975 \\
\hline 240 & 2.48704 & 1.475497 & 0.1037893 & 10.9671 \\
\hline 250 & 2.452946 & 8.380901 & -0.1970856 & 7.226189 \\
\hline 260 & 2.205224 & 11.06502 & -0.4807954 & -0.1544821 \\
\hline 270 & 1.765449 & 8.175891 & -0.7226327 & -7.457364 \\
\hline 280 & 1.171921 & 1.168729 & -0.9015357 & -11.00408 \\
\hline 290 & 0.4763283 & -6.427075 & -1.001924 & -9.008176 \\
\hline 300 & -0.2607465 & -10.78558 & -1.015054 & -2.474989 \\
\hline
\end{tabular}

TABle 2: The numerical solutions $p_{2}, \gamma_{2}$, and their derivatives.

\begin{tabular}{lcccc}
\hline$t$ & $p_{2}$ & $\gamma_{2}$ & $\dot{p}_{2}$ & $\dot{\gamma}_{2}$ \\
\hline 0 & 1.5 & 11.06602 & 0.8164966 & $8.60977 E-05$ \\
10 & 2.018441 & 8.279626 & 0.6022035 & -7.341455 \\
20 & 2.361096 & 1.324263 & 0.3354649 & -10.9858 \\
30 & 2.498125 & -6.297489 & 0.03951085 & -9.098105 \\
40 & 2.417592 & -10.74771 & -0.2598841 & -2.629244 \\
50 & 2.126513 & -9.78573 & -0.5366458 & 5.163152 \\
60 & 1.650237 & -3.896276 & -0.7666712 & 10.3552 \\
70 & 1.030242 & 3.954753 & -0.9299275 & 10.33263 \\
80 & 0.3205241 & 9.813907 & -1.012197 & -107155 \\
90 & -0.4171081 & 10.73098 & -1.006314 & -2.68967 \\
100 & -1.118414 & 6.244486 & -0.9127917 & -9.131646 \\
110 & -1.722318 & -1.38609 & -0.739775 & -10.97505 \\
120 & -2.176226 & -8.318236 & -0.5023316 & -7.291935 \\
130 & -2.440607 & -11.06137 & -0.2211403 & 0.06275124 \\
140 & -2.492436 & -8.234457 & 0.07930994 & 7.385382 \\
150 & -2.3272 & -1.261325 & 0.372853 & 10.9887 \\
160 & -1.959289 & 6.346504 & 0.6339244 & 9.058519 \\
170 & -1.420744 & 10.75812 & 0.8397874 & -5.567106 \\
180 & -0.7584675 & 9.752295 & 0.9725135 & -10.37296 \\
190 & -0.03013661 & 3.835833 & 1.020544 & -10.30583 \\
200 & 0.7008187 & -4.011765 & 0.9796954 & -5.049277 \\
210 & 1.37074 & -9.83878 & 0.8535257 & \\
\hline
\end{tabular}


TABLE 2: Continued.

\begin{tabular}{lcccc}
\hline$t$ & $p_{2}$ & $\gamma_{2}$ & $\dot{p}_{2}$ & $\dot{\gamma}_{2}$ \\
\hline 220 & 1.921283 & -10.71119 & 0.6530228 & 2.749476 \\
230 & 2.304503 & -6.190003 & 0.3956484 & 9.163264 \\
240 & 2.487025 & 1.44783 & 0.1038172 & 10.96256 \\
250 & 2.452952 & 8.356145 & -0.1970554 & 7.24163 \\
260 & 2.205252 & 11.05635 & -0.4807664 & -0.1255394 \\
270 & 1.765499 & 8.189053 & -0.7226074 & -7.429035 \\
280 & 1.171988 & 1.198392 & -0.9015169 & -10.99124 \\
290 & 0.4764102 & -6.395274 & -1.001913 & -9.018668 \\
300 & -0.2606581 & -10.76818 & -1.015054 & -2.504935 \\
\hline
\end{tabular}

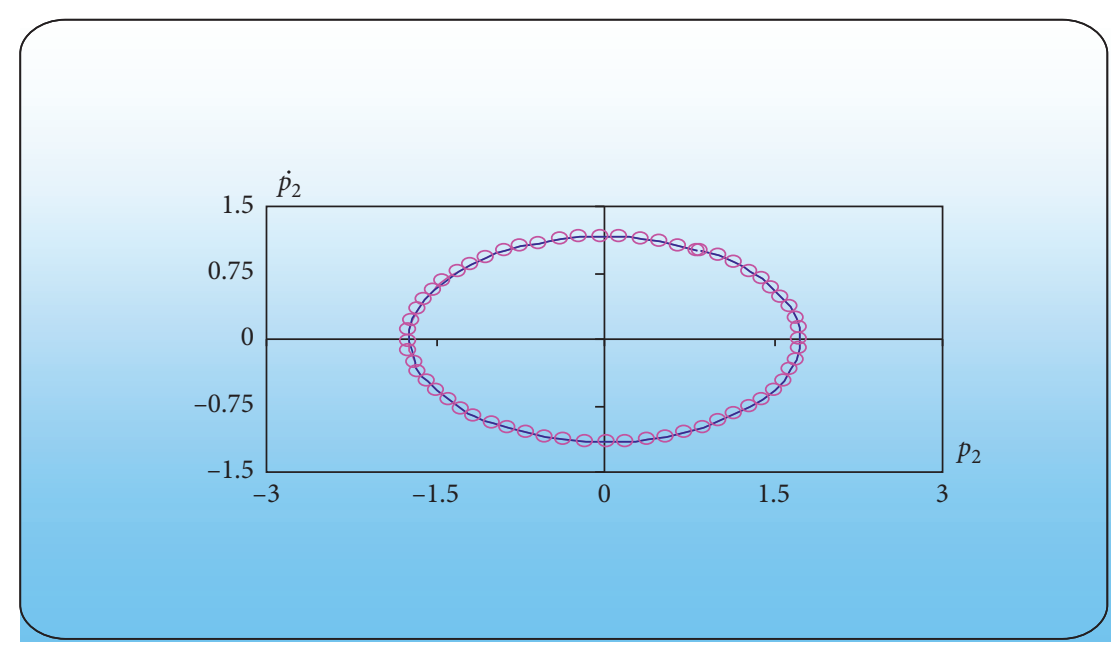

FIgURE 3: The stability of the analytical and numerical solutions $\dot{p}_{2}$ and $p_{2}$.

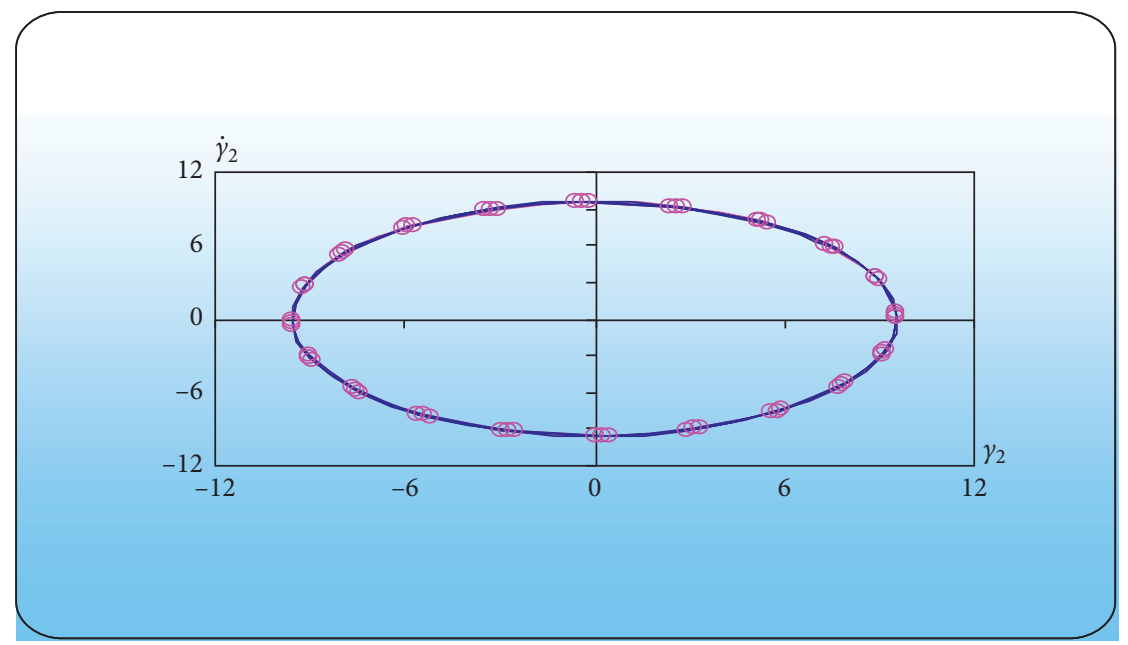

FIGURE 4: The stability of the analytical and numerical solutions $\dot{\gamma}_{2}$ and $\gamma_{2}$.

\section{The Numerical Solutions}

In this section, we assume numerical values data for the parameters of a rigid body, and we achieve a computer program to solve the quasilinear system using the fourth order Runge-Kutta method [7]. We make another program to represent the analytical solutions numerically in a period $t$ between 0 and
300 (Table 1). We use the initial values from Table 1 for obtaining the numerical solutions represented in Table 2. The comparison between the obtained numerical solutions and analytical ones is presented to know the difference between them. The numerical and analytical solutions are in good agreement with others which proves the accuracy of used methods and obtained results. 


\section{Conclusion}

The solutions (46) and the correction of the period (47) are obtained using the large parameter method, which had never been used for solving this kind of problem in the presence of the new assumptions for motion (the weak oscillations of the body about the minor or the major axis of the ellipsoid of inertia instead of the strong oscillations in the previous works). The advantage of this method is that the energy motion of the body is assumed to be sufficiently small instead of sufficiently large with other techniques [10-12]. Also, the obtained solutions treat a singular situation for the natural frequency which was excluded from previous works $[13,14]$.

Equations (50) and (51) describe the rotation of the body at any time and show that this motion depends on four arbitrary constants $\xi_{0}, \zeta_{0}, \eta_{0}$, and $r_{0}$, such that $r_{0}$ is sufficiently small. The obtained solutions give special cases of motions when $\left(M_{1}=M_{2}=0\right)$ and when $M_{1}=0, M_{2} \neq 0$, or $M_{2}=0, M_{1} \neq 0$. Also, the obtained solutions give many gyroscopic motions, which depend on the values of the moments of inertia and the initial position of the body center of gravity. In the end, we obtain the case of regular precession $[10]$ as a special case.

The analytical solutions (46) are represented indefinite intervals of time through computer programs (Table 1). The numerical solutions are obtained using the fourth order Runge-Kutta method in terms of another program (Table 2). Tables 1 and 2 give in detail the obtained results of both the analytical solutions and numerical ones. These results show that the analytical solutions are in full agreement with the numerical ones which proves the accuracy of the considered techniques and results. This case of study is considered as a general case of such ones studied in [5]. The stability phase diagrams of the solutions $p_{2}$ and $\gamma_{2}$ are given (Figures 3 and 4). From these diagrams, we note that the stability for both the analytical and the numerical solutions in full agreement. This gives the validity of the obtained solutions and the considered procedures. The considered procedures and results are very useful for the general reader's concern with the new applications dealing with the use of functionally graded materials in such structures based on the recent works [15].

\section{Data Availability}

Data sharing is not applicable to this article as no datasets were generated or analyzed during the current study.

\section{Conflicts of Interest}

The authors declare that they have no conflicts of interest.

\section{References}

[1] F. E. Udwadia and R. E. Kalaba, Analytical Dynamics: A New Approach, Cambridge University Press, Cambridge, UK, 2007.

[2] T. S. Amer, A. I. Ismail, and W. S. Amer, "Application of the Krylov-Bogoliubov-Mitropolski technique for a rotating heavy solid under the influence of a gyrostatic moment,"
Journal of Aerospace Engineering, vol. 25, no. 3, pp. 421-430, 2012.

[3] A. I. Ismail, "On the motion of a rigid body in a Newtonian field of force exerted by three attractive centers," Journal of Aerospace Engineering, vol. 21, no. 1, pp. 67-77, 2011.

[4] A. H. Nayfeh, Introduction to Perturbation Technique, Wiley, Hoboken, NJ, USA, 2011.

[5] A. I. Ismail, "Solving a problem of rotary motion for a heavy solid using the large parameter method," Advances in Astronomy, vol. 2020, Article ID 2764867, 7 pages, 2020.

[6] S. V. Ershkov and D. Leshchenko, "On a new type of solving procedure for Euler-Poisson equations (rigid body rotation over the fixed point)," Acta Mechanica, vol. 230, no. 3, pp. 871-883, 2019.

[7] C. T. Wu, L. Wang, B. Bonello, L. Ling, N. Ma, and M. A. Schweitzer, "Advanced Mesh-based and particle-based numerical methods for engineering and applied mathematics problems," Mathematical Problems in Engineering, vol. 2017, Article ID 1273017, 2 pages, 2017.

[8] A. I. Ismail and T. S. Amer, "A necessary and sufficient condition for solving a rigid body problem," Technische Mechanik, vol. 31, no. 1-2, pp. 50-57, 2011.

[9] L. D. Akulenko, Y. S. Zinkevich, T. A. Kozachenko, and D. D. Leshchenko, "The evolution of the motions of a rigid body close to the Lagrange case under the action of an unsteady torque," Journal of Applied Mathematics and Mechanics, vol. 81, no. 2, pp. 79-84, 2017.

[10] A. Sanders Jan, V. Ferdinand, and M. James, "Averaging methods in nonlinear dynamical systems," Applied Mathematical Sciences, vol. 59, no. 2, p. 434, 2007.

[11] F. L. Chernousko, L. D. Akulenko, and D. D. Leshchenko, Evolution of Motions of a Rigid Body about Its Center of Mass, Springer International Publishing, Berlin, Germany, 2017.

[12] A. I. Ismail and F. D. El-Haiby, "Torque free axi-symmetric gyros with changing moments of inertia," Applied Mathematics, vol. 7, no. 16, p. 1934, 2016.

[13] T. S. Amer, "On the dynamical motion of a gyro in the presence of external forces," Advances in Mechanical Engineering, vol. 9, no. 2, pp. 1-13, 2017.

[14] T. S. Amer and I. M. Abady, "On the motion of a gyro in the presence of a Newtonian force field and applied moments," Mathematics and Mechanics of Solids, vol. 23, no. 9, pp. 1263-1273, 2017.

[15] N. Bendenia, M. Zidour, and A. A. Bousahla, "Deflections, stresses and free vibration studies of FG-CNT reinforced sandwich plates resting on Pasternak elastic foundation," Computers and Concrete, vol. 26, no. 3, pp. 213-226, 2020. 